\title{
Analysis of a fast-acting waveguide ferrite phase shifter with longitudinal magnetization phasing structure
}

\author{
Elena V. Komissarova ${ }^{1, *}$, Vladimir M. Krekhtunov ${ }^{1}$, and Yury S. Rusov ${ }^{1}$ \\ ${ }^{1}$ Bauman Moscow State Technical University, 5 building 1, $2^{\text {nd }}$ Baumanskaya str., Moscow, 105005, \\ Russia
}

\begin{abstract}
The electrodynamic analysis and calculation of the phasing structure of a waveguide ferrite Faraday phase shifter with longitudinal magnetization of a ferrite rod with a square cross-section are performed. In the strict electrodynamic formulation, the Galerkin method solves the key problem for the design of the phase shifter: the problem of eigenwaves finding with a second-order differential magnetic operator in the projection procedure for a rectangular waveguide with a transversely inhomogeneous ferrite-dielectric filling under longitudinal magnetization. The problem is reduced to solving the complete eigenvalue problem of a complex matrix. The computational algorithm, implemented as a computer program in DELPHI, gives in one procedure the numerical values of the coefficients of the decomposition of fields in the form of eigenvectors of the matrix, and the coefficients of wave propagation in the form of its eigenvalues. The results of calculations of the phasing structure activity for two particular cases of its implementation are presented: on the basis of a ferrite rod both with a conductive coating and without a coating, it is shown that in the second case the activity of the phase shifter is 1.5 times higher. For the real parameters of the ferrite medium, the length of the phasing structure is calculated depending on the transverse dimensions of the ferrite rod and the square waveguide, which ensures the creation of a $360^{\circ}$ controlled phase shift.
\end{abstract}

\section{Introduction}

Waveguide Faraday ferrite phase shifters (WFFPS) with longitudinal magnetization are widely used in the microwave and EHF bands technique, both as standalone devices in waveguides $[1,2]$, and as a part of devices, for example, elements of integrated phased antenna arrays (PAA) $[3,4,5,6]$. As a rule, these WFFPS are performed on the basis of ferrite rods (FR) with a round or square cross-sectional shape $[4,5,6]$. The most commonly used devices are those with FR having a conductive coating on the side surface [3,4]. These WFFPS structurally simple and easy to manufacture. However, they have such disadvantages as low activity, low shifting speed and high shifting energy of a FR from one phase state to another. This is a consequence of the excitation of Foucault currents in the conductive coating of the FR at its pulsed remagnetization.

${ }^{*}$ Corresponding author: $\underline{\text { mc_ken@list.ru }}$ 
WFFPS, which is built on the basis of the FR, which does not have a conductive coating of the side surface, is free from these disadvantages [5, 6, 7]. Such WFFPS has the longitudinal magnetization system and the magnetic core providing device magnetic memory are placed inside a waveguide. This leads to a decrease in Foucault currents, the magnetization reversal time and the control energy of the WFFPS [7]. At the same time, the activity and q-factor of the phase shifter increases, and so its longitudinal dimensions reduce. However, while using a phase shifter with magnetic memory based on anonmetallized ferrite rod, i.e. without a conductive coating of its side surface, the device becomes multimode, its calculation becomes more complicated and there is a need for highlevel electrodynamic models.

\section{Object and research methods}

In the article the phasing structures of WFFPS based on square waveguides partially or completely filled with rectangular ferrite-dielectric rods is analized. On Fig. 1 is shown a longitudinal section of WFFPS comprising waveguide 1 , matching transitions 2 , ferrite rod 3 , the longitudinal magnetization with the frame 4 , ferrite yoke 5 , which pads are adjacent to the side ferrite rod surface, providing a "magnetic memory" of WFFPS.

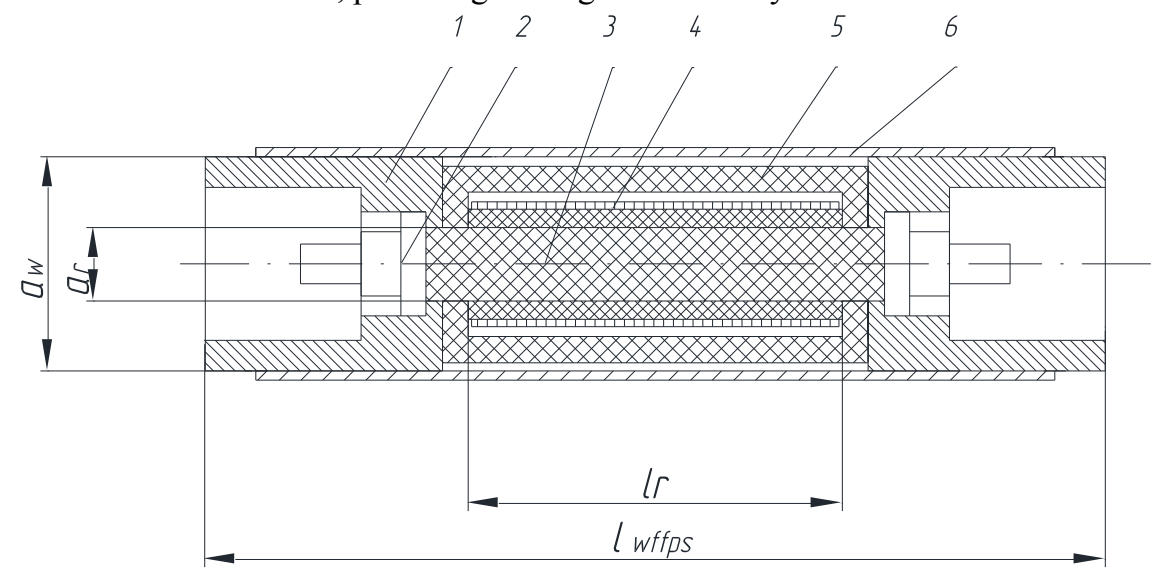

Fig. 1. Waveguide ferrite Faraday phase shifter draft.

Using Galerkin method [7] with strict electrodynamic formulation the key problem of WFFPS design - the problem of eigenwaves finding of the for a rectangular waveguide with transverse inhomogeneous ferrite-dielectric longitudinal magnetized filling the is solved. On Fig. 2 the cross sections of the under study waveguides is shown.

On Fig. $2 \mathrm{a}$ is the general case when the inside filling of the waveguide is characterized by a dielectric permeability $\varepsilon_{\mathrm{a}}(\mathrm{x}, \mathrm{y})=\varepsilon_{0} \cdot \varepsilon(\mathrm{x}, \mathrm{y})$ and magnetic permeability tensor $\overline{\bar{\mu}}_{a}(x, y)=\mu_{0} \cdot \overline{\bar{\mu}}(x, y)$, where $\varepsilon(\mathrm{x}, \mathrm{y})$ и $\mu_{\mathrm{i}}(\mathrm{x}, \mathrm{y})-$ piecewise continuous functions of transverse coordinates.

For harmonic oscillations with the frequency $\omega$ need to solve the system of Maxwell's equations [7]

$$
\left\{\begin{array}{l}
\operatorname{rot} \vec{H}=j \omega \varepsilon_{a} \vec{E} ; \quad \operatorname{div} \varepsilon_{a} \vec{E}=0 ; \\
\operatorname{rot} \vec{E}=-j \omega \overline{\bar{\mu}}_{a} \vec{H} ; \quad \operatorname{div} \overline{\bar{\mu}}_{a} \vec{H}=0 .
\end{array}, \overline{\bar{\mu}}(x, y)=\left\|\begin{array}{ccc}
\mu_{1} & -j \mu_{2} & 0 \\
j \mu_{2} & \mu_{1} & 0 \\
0 & 0 & \mu_{3}
\end{array}\right\|,\right.
$$




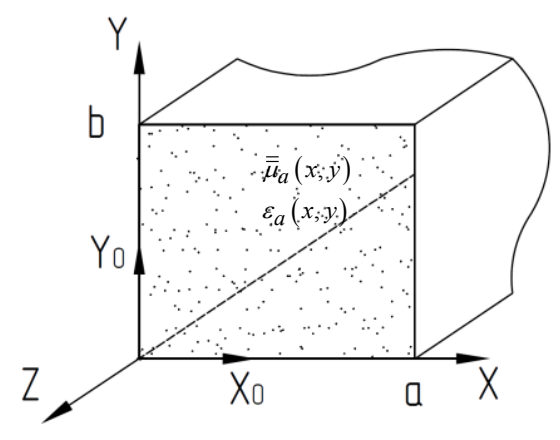

$\mathrm{a}$

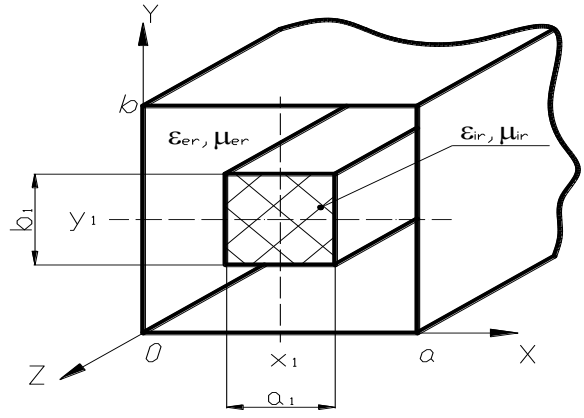

b

Fig. 2. Waveguides with transversely inhomogeneous ferrite-dielectric filling.

After the vector $\vec{E}$ and component $\mathrm{H}_{\mathrm{z}}$ are excluded from the system of equations (1), the problem is reduced to solving a system of differential equations ob the transverse vector of the magnetic field $\vec{H}_{\perp}(x, y)$ [10]

$$
L_{H}^{\mu} \vec{H}_{\perp}(x, y)=p^{2} k_{0}^{2} \vec{H}_{\perp}(x, y),
$$

where $L_{H}^{\mu}-$ second-order differential magnetic operator [10], which elements together with $\varepsilon_{\mathrm{i}}(\mathrm{x}, \mathrm{y})$ and $\mu_{\mathrm{i}}(\mathrm{x}, \mathrm{y})$, consists of their transverse coordinates derivatives $\partial / \partial \mathrm{x}$ и $\partial / \partial \mathrm{y}$; $k_{0}=\omega \sqrt{\varepsilon_{0} \mu_{0}}, \beta$ - phase coefficient of eigenwaves and $\mathrm{p}=\beta / \mathrm{k}_{0}$ - wave deceleration rate.

When solving the operator equation (2) by the Galerkin method [7], the required field $\vec{H}_{\perp}(x, y)$ is represented as an expansion

$$
\vec{H}_{\perp}(x, y)=\sum_{t=1}^{2} \sum_{m=0}^{\infty} \sum_{n=0}^{\infty} M_{t m n} \vec{h}_{t m n}(x, y), m+n \geq 1,
$$

here $\mathrm{M}_{\mathrm{tmn}}$ - the coefficients of the fields expansion of their own waves of a waveguide with transverse inhomogeneous lling in the system of rectangular waveguide transverse vector functions $\vec{h}_{t m n}(x, y)$, index $\mathrm{t}=1,2$ corresponds to the waves of rectangular waveguide $\mathrm{H}_{\mathrm{mn}}$ and $\mathrm{E}_{\mathrm{mn}}$ types.

In accordance with the Bubnov-Galerkin procedure [7] the required field (3) is substituted into the operator equation (2) and the equation is found as

$$
L_{H}^{\mu} \sum_{t=1}^{2} \sum_{m=0}^{\infty} \sum_{n=0}^{\infty} M_{t m n} \vec{h}_{t m n}(x, y)=p^{2} k_{0}^{2} \sum_{t=1}^{2} \sum_{m=0}^{\infty} \sum_{n=0}^{\infty} M_{t m n} \vec{h}_{t m n}(x, y) .
$$

After projecting equation (4) on a system of basic vector functions $\vec{h}_{t m n}(x, y)$ taking into account their orthonormality, the problem is reduced to solving the complete eigenvalue problem of the complex matrix

$$
\mathbf{A M}=p^{2} \mathbf{M}
$$

Its elements could be written as 


$$
\begin{aligned}
& A_{t m n}^{t^{\prime} m^{\prime} n^{\prime}}=N_{t m n} N_{t^{\prime} m^{\prime} n^{\prime}}\left(\xi_{t m n 1} \xi_{t^{\prime} m^{\prime} n^{\prime} 1} I_{m^{\prime} n^{\prime} m n}^{(1)}+\xi_{t m n 2} \xi_{t^{\prime} m^{\prime} n^{\prime} 2} I_{m^{\prime} n^{\prime} m n}^{(2)}\right)+ \\
& +\left[\chi_{m}\left(\xi_{t m n 2} / k_{0}\right)-\chi_{n}\left(\xi_{t m n 1} / k_{0}\right)\right] N_{t m n} N_{t^{\prime} m^{\prime} n^{\prime} n^{\prime}}\left(\xi_{t^{\prime} m^{\prime} n^{\prime} 2} I_{m^{\prime} n^{\prime} m n}^{(3)}+\xi_{t^{\prime} m^{\prime} n^{\prime} 1^{\prime}} I_{m^{\prime} n^{\prime} m n}^{(4)}\right)- \\
& -\left(\chi_{m}^{2}+\chi_{n}^{2}\right) \delta_{t^{\prime} t} \delta_{m^{\prime} m} \delta_{n^{\prime} n} .
\end{aligned}
$$

Elements could be reduced to the solution of integrals

$$
\left\{\begin{array}{l}
I_{m^{\prime} n^{\prime} m n}^{(1)}=\int_{S} \varepsilon \cdot \overline{\bar{\mu}}_{1} \cdot \sin \left(u_{m} x\right) \cdot \cos \left(u_{n} y\right) \cdot \sin \left(u_{m^{\prime}} x\right) \cdot \cos \left(u_{n^{\prime}} y\right) d s ; \\
I_{m^{\prime} n^{\prime} m n}^{(2)}=\int_{S} \varepsilon \cdot \overline{\bar{\mu}}_{2} \cdot \cos \left(u_{m} x\right) \cdot \sin \left(u_{n} y\right) \cdot \cos \left(u_{m^{\prime}} x\right) \cdot \sin \left(u_{n^{\prime}} y\right) d s ; \\
I_{m^{\prime} n^{\prime} m n}^{(3)}=\int_{S}(1 / \varepsilon) \cdot(\partial \varepsilon / \partial x) \cdot \sin \left(u_{m} x\right) \cdot \sin \left(u_{n} y\right) \cdot \cos \left(u_{m^{\prime}} x\right) \cdot \sin \left(u_{n^{\prime}} y\right) d s ; \\
I_{m^{\prime} n^{\prime} m n}^{(4)}=\int_{S}(1 / \varepsilon) \cdot(\partial \varepsilon / \partial y) \cdot \cos \left(u_{m} x\right) \cdot \sin \left(u_{n} y\right) \cdot \cos \left(u_{m^{\prime}} x\right) \cdot \sin \left(u_{n^{\prime}} y\right) d s ;
\end{array}\right.
$$

Here $\delta$ - Kronecker symbol, $u_{m}=(m \pi / a) ; u_{n}=(n \pi / b)$, coefficients $\mathrm{M}_{\mathrm{tmn}}$ are identified with the numerical values of the eigenvectors of the matrix $\mathbf{A}$, the squares of the waves slowing coefficients $\mathrm{p}^{2}-$ with its eigenvalues.

For some special cases of a waveguide with ferrite-dielectric filling, shown in Fig. 1, i.e. for given functions $\varepsilon_{\mathrm{i}}(\mathrm{x}, \mathrm{y})$ and $\mu_{\mathrm{i}}(\mathrm{x}, \mathrm{y})$, the integrals appearing in the expressions for the calculation of the matrix elements are reduced to a table, and formulas for their calculation are explicitly.

\section{Numerical results}

The main part of the WFFPS is a phasing block, the ferrite rod cross-sectional dimensions of which are calculated and optimized: its "active" length $1_{1}$ (Fig. 1) and ferrite rod crosssection size $a_{r}$. The choice of the transversal dimensions is determined by the conditions of cross-sectional dimension of WFFPS minimization and the length of the FR that is needed to create the desired differential insertion phase shift $\Delta \varphi_{\max }$. Active part length of the FR is calculated by the formula

$$
\Delta \varphi_{\max }=k \cdot l_{1} \cdot \Delta p, l_{1}=\frac{\Delta \varphi_{\max }}{2 \pi} \cdot \frac{\lambda_{0}}{\Delta p} .
$$

где $\Delta \mathrm{p}=\mathrm{p}^{(+)}-\mathrm{p}^{(-)} ; \mathrm{p}^{(+)}$и $\mathrm{p}^{(-)}$- waves slowing coeffitients of ferrite rod with square cross-size.

The calculations are performed for ferrites 1SCH4 and 1SCH12 [9] used in the microwave-range. Values of $\mu_{2}$, dispersion characteristics of its own waves in a wide range of parameters were calculated for two ferrite grades according to their parameters $a_{\mathrm{r}} / \lambda$, $a_{\mathrm{w}} / \lambda ; a_{\mathrm{r}} / a_{\mathrm{w}}$.

Due to necessitate of the WFFPS based on metallized ferrite rod design the dispersion characteristics (Fig. 3) for waveguide with a square cross-size completely and uniformly filled with longitudinally magnetized ferrite (Fig. 2a) were calculated. 


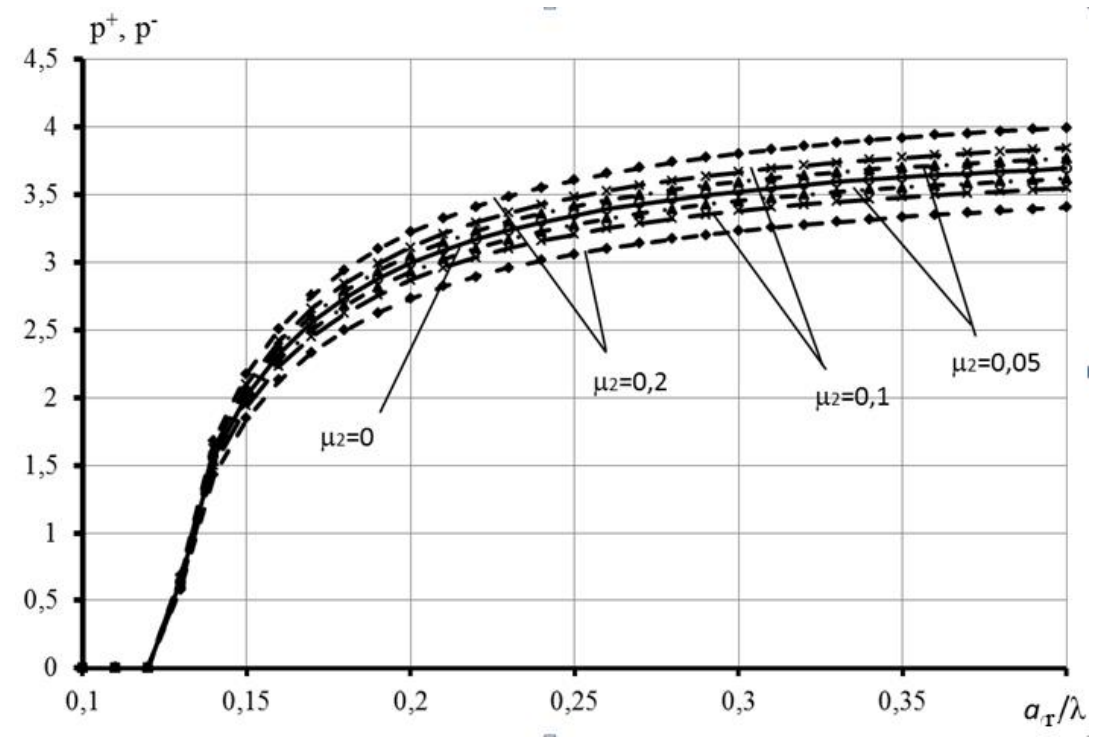

Fig. 3. Waves slowing coeffitients on parameter $a_{\mathrm{r}} / \lambda$ functions at different values of magnetization $\mu_{2}$.

In such type of waveguide circle polarized waves $\mathrm{H}_{10}{ }^{(+)}$и $\mathrm{H}_{10}{ }^{(-)}$propagate. Functions $\mathrm{p}^{(+)}\left(\mathrm{a}_{\mathrm{r}} / \lambda\right)$ and $\mathrm{p}^{(-)}\left(\mathrm{a}_{\mathrm{r}} / \lambda\right)$ were calculated with a meanings of relative dielectric permittivity $\varepsilon_{\mathrm{r}}=15,2$ and non-diagonal component of the magnetic permeability tensor $\mu_{2}=0 ; 0,1 ; 0,2$. If $\mathrm{a}_{\mathrm{r}} / \lambda$ unlimitedly increase the waves would degenerate into flat circle-polarized waves of free space and their deceleration coefficients would have the same value as counted by formula

$$
p^{( \pm)}=\sqrt{\varepsilon_{r}\left(\mu_{1} \pm \mu_{2}\right)} .
$$

On Fig. 4 calculated from the found values $\mathrm{p}^{(+)}$and $\mathrm{p}^{(-)}$functions of phase structure activity $\Delta \mathrm{p}\left(a_{\mathrm{r}} / \lambda\right)$ (Fig. $4 \mathrm{a}$ ) and ferrite rod «active» length $l_{\mathrm{r}}\left(a_{\mathrm{r}} / \lambda\right)$ (Fig. $\left.4 \mathrm{~b}\right)$ for $\Delta \varphi_{\max }=2 \pi$ are shown/
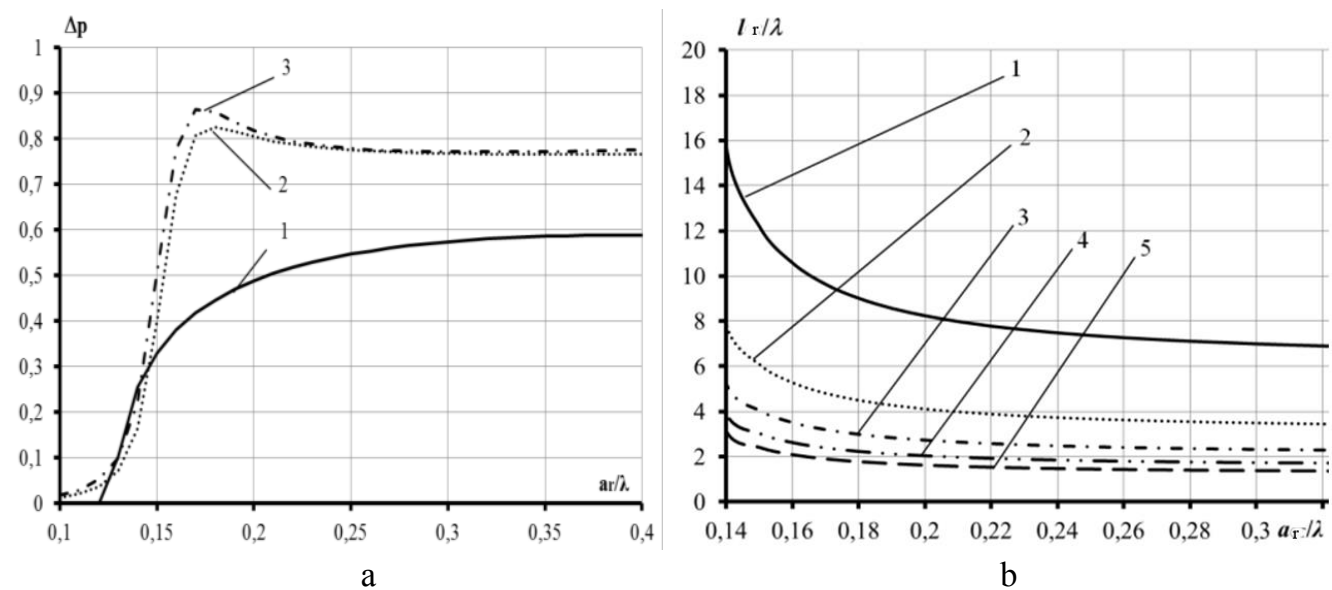

Fig. 4. Functions of phase structure activity $\Delta \mathrm{p}\left(a_{\mathrm{r}} / \lambda\right)$ while $\mu_{2}=0,15$ (a) and length $l_{\mathrm{r}}\left(a_{\mathrm{r}} / \lambda\right)$ with different numbers of $\mu_{2}$ (b). 
Lines 2 and 3 on Fig. 4a were calculated for the ferrite rod with sqare form of crosssection sizes' $\mathrm{a}^{2}$, which is placed coaxially to the waveguide with square cross-section $\mathrm{a}_{\mathrm{w}}{ }^{2}$, when $a_{w}=0,5 \lambda$ (Line 2) and $a_{w}=1,0 \lambda$ (Line 3). Of those shown in Fig. $4 \mathrm{~A}$ data suggest that the activity of WFFPS based on FR without conductive coating of the side surface in 1.5 times higher of the activity of WFFPS, based on FR with a conductive coating side surface (line 1) while $a_{\mathrm{r}} / \lambda \leq 0,25$. Consequently, the WFFPS based on FR without conductive coating could have a shorter length and lower microwave losses.

\section{Summary}

1. In the strict electrodynamic formulation by the Galerkin method using a second-order differential magnetic operator in the projection procedure, the key problem for the development of the WFFPS - the problem of eigenwaves of a waveguide with a transversely inhomogeneous ferrite-dielectric filling under longitudinal magnetization is solved.

2. The computational algorithm, implemented as a computer program in DELPHI, in one procedure gives the numerical values of the coefficients of the fields decomposition in the form of eigenvectors of the matrix, and the coefficients of wave propagation in the form of eigenvalues of the matrix, the elements of which are found explicitly

3. Calculated in a wide range of changes of transverse dimensions of the waveguides and the ferrite rod material and ferrite parameters the dispersion characteristics of the lower natural waves of the waveguide with coaxially longitudinally magnetized square crosssection ferrite rod and the completely filled waveguide with longitudinally magnetized ferrite can serve as a basis for designing WFFPS on the basis of these waveguide structures.

\section{References}

1. A.L. Mikaelyan, Theory and practice of microwave ferrites. 664 pp. (1963)

2. Boyd C.R. Comments on the design and manufacture of dual-mode reciprocal latching ferrite phase shifters // IEEE Trans. on MTT. 6. P. 593-601 (1974)

3. Yu.N. Afanasev, V.V. Zhigarev, K.n. Zakharev, V.A. Kashin, V,M, Koretsky, A.A. Lemansky, Patent 2184410 Russia. Receiving-Transmitting Phased Array Antenna Element (2002)

4. L.B. Roshal, A.I. Firsenkov, V.M. Krekhtunov, O. Yu. Shevtsov, Patent 2325741 Russia. Phased Array Antenna Element (2008)

5. E.V. Komissarova, V.M. Krekhtunov, Patent 2474018 Russia. Phased Reflective Array Antenna Element (2013)

6. A.I. Firsenkov, V.M. Krekhtunov, A.B. Guskov, G.D. Pavlov, Yu.S. Rusov, Patent 2439759 Russia. Phased Array Antenna Element (2010)

7. V.V. Nikolsky, Projection methods in electrodynamics. 1. P. 4-50. (1977)

8. A.I. Sinany, E.I. Starshinova, A.Ye. Chalykh, Patent 2379799 Russia. Microwave phase shifter (2010)

9. Microwave Ferrites and Dielectrics: http://www.magneton.ru/cat.php?id=32 (2019)

10. E.V. Komissarova Research and design of waveguide ferrite Faraday phase shifters and PAA elements based on them for the shortwave part of the millimeter wave range: $\mathrm{PhD}$ dissertation thesis $16 \mathrm{pp}$ (2015) 